Z Rheumatol 2019 · 78:955-966

https://doi.org/10.1007/s00393-019-00687-0

Online publiziert: 29. August 2019

(c) Springer Medizin Verlag GmbH, ein Teil von Springer Nature 2019

\section{Redaktion}

M. Fleck, Bad Abbach

\section{B. Hellmich · C. Löffler}

Vaskulitiszentrum Süd, Klinik für Innere Medizin, Rheumatologie und Immunologie, Medius Kliniken Akademisches Lehrkrankenhaus, Universität Tübingen, Kirchheim u. Teck, Deutschland

\title{
Was sind die Indikationen für Rescue-Verfahren?
}

\section{Rheumatische Systemerkrankungen auf der Intensivstation}

Bis zu ein Drittel aller Patienten mit einer entzündlich-rheumatischen Erkrankung, die als Notfall in ein Akutkrankenhaus aufgenommen werden, benötigt im Lauf des stationären Aufenthaltes eine intensivmedizinische Überwachung und/oder Therapie [27, 54]. Ergebnisse einer systematischen Literaturanalyse ergaben, dass bis zum Jahr 2000 die rheumatoide Arthritis (RA) als häufigste Autoimmunerkrankung auf einer Intensivstation gefunden wurde. Seit dem Jahr 2000 haben der systemische Lupus erythematodes (SLE) gefolgt von den systemischen Vaskulitiden die RA als häufigste rheumatologische Diagnosen auf der Intensivstation abgelöst [54], am ehesten bedingt durch die verbesserten Therapiemöglichkeiten mit der Einführung der Biologika kurz vor der Jahrhundertwende. Die Mortalität von Patienten mit systemischen Autoimmunerkrankungen auf der Intensivstation ist hoch und reicht in den verschiedenen Fallserien von ca. $17-55 \%$ und ist tendenziell höher, wenn eine Infektion der führende Anlass für eine Aufnahme auf die Intensivstation darstellt [12, 54]. Bei Patienten mit rheumatischen Systemerkrankungen stellen in etwa gleicher Häufigkeit Infektionen und schwere pulmonale Manifestationen (diffuse alveoläre Hämorrhagie, fortgeschrittene interstitielle Lungenerkrankung, pulmonalarterielle Hypertonie etc.) die häufigsten Gründe für eine Aufnahme auf die Intensivstation dar, gefolgt von anderen schweren Organkomplikationen als Folge einer entzündlichen Aktivität der Grunderkrankung (z.B. schneller
Nierenfunktionsverlust bei rapid-progressiver Glomerulonephritis, kardiale Beteiligung, schweres Makrophagenaktivierungssyndrom etc.) $[10,54]$.

Insbesondere bei Intensivpatienten ist die Unterscheidung zwischen einer entzündlichen Aktivität der Grunderkrankung und einer Infektion nicht trivial. Neben einem kompetenten infektiologischen Management ist eine schnell wirksame Kontrolle der entzündlichen Aktivität der Grunderkrankung für die individuelle Prognose des Patienten von zentraler Bedeutung. Aufgrund ihres raschen Wirkeintritts stellen hoch dosierte Glukokortikoide (GC) die Behandlung der ersten Wahl bei organoder lebensbedrohlichen Manifestationen von Kollagenosen, Vaskulitiden und anderen systemischen Autoimmunerkrankungen dar [21, 60, 74]. In der Regel wird die Therapie mit GC bei kritischen Manifestationen um potente konventionelle Immunsuppressiva (insbesondere Cyclophosphamid) und/oder Biologika ergänzt (s. hierzu auch den Beitrag von F. Buttgereit in dieser Ausgabe). Die Therapie mit diesen Substanzen verbessert im Vergleich zu einer GCMonotherapie langfristig die Prognose [25], die Wirkung setzt im Vergleich zu GC jedoch mit einer Latenz von mehreren Tagen bzw. Wochen ein und ist daher für die kurzfristige Beherrschung eines bedrohlichen Krankheitsschubes (z. B. einer diffusen alveolären Hämorrhagie) noch zu schwach. Zudem erhöht die sehr intensive Immunsuppression in Verbindung mit den häufig erforderlichen intensivmedizinischen
Maßnahmen (Beatmung, zentrale Venenkatheter) in dieser Situation das Risiko für schwere Infektionskomplikationen während des intensivstationären Aufenthaltes. Für die Wirksamkeit einer immunsuppressiven Therapie einiger Akutkomplikationen wie rezidivierende Thrombembolien im Rahmen eines "katastrophalen“" Antiphospholipidsyndroms (APS) liegt nur eine allenfalls schwache Evidenz vor [37]. Es besteht daher nicht selten die Notwendigkeit, die immunsuppressive Therapie auf der Intensivstation um rasch wirksame oder ggf. auch weniger immunsuppressiv wirksame Rescue-Therapieverfahren zu ergänzen oder zu ersetzen. Diese Übersichtsarbeit soll einen Überblick über Rescue-Therapieverfahren mit nicht primär immunsuppressivem Wirkprinzip geben und dabei insbesondere den Stellenwert der Plasmapherese (• Tab. 1), der extrakorporalen Membranoxygenierung $($ ECMO) (• Tab. 2) und der Therapie mit intravenösen Immunglobulinen (IVIG) (- Tab. 3) beleuchten. Das Evidenzniveau wurde gemäß der Einteilung des Oxford Center of Evidence-based Medicine bewertet [46].

\section{Plasmapherese}

Die Plasmapherese ist ein mechanisches Verfahren, das Plasmabestandteile mit hohem Molekulargewicht entfernt. Die Rationale zum Einsatz der Plasmapherese als Rescue-Therapieverfahren besteht in der raschen Auswaschung von für die Pathogenese von Autoimmunerkrankungen relevanten Molekülen [13]. Zu den 


\section{Leitthema}

Tab. 1 Indikationen zur Plasmapherese bei kritisch kranken Patienten mit entzündlich-rheumatischen Erkrankungen

\begin{tabular}{|c|c|c|c|c|c|}
\hline Erkrankung & \multicolumn{2}{|l|}{ Indikation } & \multicolumn{2}{|l|}{ Evidenzgrad $^{a}$} & Kommentar \\
\hline \multirow{2}{*}{$\begin{array}{l}\text { Anti-GBM- } \\
\text { Erkrankung }\end{array}$} & \multicolumn{2}{|c|}{ Akute Glomerulonephritis } & \multirow{2}{*}{\multicolumn{2}{|c|}{$2 b$}} & First-Line-Therapie mit CYC und GC \\
\hline & \multicolumn{2}{|c|}{ Diffuse alveoläre Hämorrhagie } & & & Leitlinienempfehlung (KDIGO) \\
\hline \multirow{2}{*}{$\begin{array}{l}\text { Kryoglobu- } \\
\text { linämische } \\
\text { Vaskulitis }\end{array}$} & \multicolumn{2}{|c|}{$\begin{array}{l}\text { Refraktäre schwere/lebensbedrohliche } \\
\text { Vaskulitis }\end{array}$} & \multicolumn{2}{|c|}{ 3b (HCV-assoziierte KV) } & Bei Versagen der Standardtherapie \\
\hline & \multicolumn{2}{|c|}{ Hyperviskositätssyndrom } & \multicolumn{2}{|c|}{4 (essenzielle KV) } & \\
\hline \multirow{3}{*}{$\begin{array}{l}\text { Katastrophales } \\
\text { APS }\end{array}$} & \multirow{3}{*}{\multicolumn{2}{|c|}{ Multiorganversagen }} & \multirow{3}{*}{\multicolumn{2}{|c|}{4}} & In Kombination mit GC und Heparin, alternativ zu IVIG \\
\hline & & & & & Stellenwert (First- oder Second-Line) kontrovers \\
\hline & & & & & Leitlinienempfehlung \\
\hline \multirow[t]{2}{*}{ SLE } & \multicolumn{2}{|c|}{$\begin{array}{l}\text { Schwere Organbeteiligung bei } \\
\text { Schwangeren }\end{array}$} & \multicolumn{2}{|l|}{5} & $\begin{array}{l}\text { Bei Kontraindikation/Versagen von GC oder anderen } \\
\text { Immunsuppressiva }\end{array}$ \\
\hline & \multicolumn{2}{|l|}{ Katastrophales APS } & \multicolumn{2}{|l|}{4} & Immunadsorption als Alternative \\
\hline \multicolumn{6}{|c|}{$\begin{array}{l}\text { GBM glomeruläre Basalmembran, CYC Cyclophosphamid, GC Glukokortikoide, KDIGO Kidney Disease: Improving Global Outcomes, APS Antiphospholi- } \\
\text { pidsyndrom, HCV Hepatitis-C-Virus, KV kryoglobulinämische Vaskulitis, IVIG intravenöse Immunglobuline, SLE systemischer Lupus erythematodes } \\
\text { aEvidenzgrad nach Oxford Center of Evidence-based Medicine } 2009[46]\end{array}$} \\
\hline \multicolumn{2}{|l|}{ Erkrankung } & Indikation & Evidenzgrad $^{\mathrm{a}}$ & \multicolumn{2}{|c|}{ Kommentar } \\
\hline \multicolumn{2}{|c|}{$\begin{array}{l}\text { Anti-GBM-Erkrankung (Goodpasture- } \\
\text { Syndrom) }\end{array}$} & $\begin{array}{l}\text { Diffuse alveoläre } \\
\text { Hämorrhagie }\end{array}$ & 4 & \multicolumn{2}{|c|}{$\begin{array}{l}\text { Bei schwerer refraktärer respiratorischer Insuffizienz trotz invasiver } \\
\text { Beatmung und immunsuppressiver Therapie }\end{array}$} \\
\hline \multicolumn{2}{|c|}{$\begin{array}{l}\text { ANCA-assoziierte Vaskulitis und ande- } \\
\text { re Kleingefäßvaskulitiden }\end{array}$} & $\begin{array}{l}\text { Diffuse alveoläre } \\
\text { Hämorrhagie }\end{array}$ & 4 & \multicolumn{2}{|c|}{$\begin{array}{l}\text { Bei schwerer refraktärer respiratorischer Insuffizienz trotz invasiver } \\
\text { Beatmung und immunsuppressiver Therapie }\end{array}$} \\
\hline \multicolumn{2}{|c|}{ Alle } & ARDS durch Infektion & $1 b$ & \multicolumn{2}{|c|}{$\begin{array}{l}\text { Bei schwerer refraktärer respiratorischer Insuffizienz trotz invasiver } \\
\text { Beatmung und antiinfektiver Therapie }\end{array}$} \\
\hline \multicolumn{6}{|c|}{$\begin{array}{l}\text { ANCA antineutrophile zytoplasmatische Antikörper, GBM glomeruläre Basalmembran, ARDS Acute-Respiratory-Distress-Syndrom } \\
\text { 'Evidenzgrad nach Oxford Center of Evidence-based Medicine } 2009 \text { [46] }\end{array}$} \\
\hline
\end{tabular}

mit einer Plasmapherese entfernbaren Molekülen zählen Autoantikörper, Komplementfaktoren und andere potenziell pathogene Substanzen wie Kryoglobuline, aber auch Gerinnungsfaktoren, Immunglobuline und andere Proteine [13, 34]. Autoantikörper wie antineutrophile zytoplasmatische Antikörper (ANCA) liegen überwiegend als Immunglobuline der Subklasse Ig(Immunglobulin)G vor [67]. Eine einzelne Plasmapheresebehandlung mit Austausch des 1,2-Fachen des individuellen Plasmavolumens pro Sitzung reduziert die IgG-Konzentration im Plasma um ca. 63\% [67]. Der überwiegende Teil des IgG liegt extravaskulär vor. Nach einer Plasmapheresesitzung dauert es ca. 1 bis 2 Tage bis ein Equilibrium zwischen intra- und extrazellulärem IgG erreicht ist. Nach insgesamt 7 Plasmaaustauschbehandlungen ist der Gesamtkörperbestand an IgG um ca. $95 \%$ reduziert [67]. Üblicherweise werden daher 7 Plasmapheresen innerhalb von 14 Tagen mit einem Austauschvolumen von $60 \mathrm{ml} / \mathrm{kgKG}$ (Körpergewicht) pro Behandlung empfohlen [71].

\section{\) Nach 7 Plasmaaus- tauschbehandlungen ist der Gesamtkörperbestand an IgG um ca. $95 \%$ reduziert}

Das im Rahmen der Plasmapherese entfernte Plasma wird (zumindest während der ersten Behandlungszyklen) durch Humanalbumin (3-5\%) ersetzt (Ausnahme ist die thrombotische thrombozytopenische Purpura [TTP]: hier primärer Austausch mit Fresh-FrozenPlasma [FFP] aufgrund der Substitution der von Willbrand-Faktor spaltenden Protease (ADAMTS13). Auch wenn durch eine Plasmapherese eine immunsuppressive Therapie theoretisch eingespart werden kann, was bei Patienten mit Infektionen oder Schwangeren vorteilhaft sein kann, handelt sich um ein potenziell komplikationsträchtiges
Verfahren: In einer prospektiven Studie kam es bei $36 \%$ aller Plasmapheresen $\mathrm{zu}$ Komplikationen, wobei die allermeisten in dieser Studie mild waren und häufig als allergische Reaktionen auf das Substituat auftraten [63]. Da die Plasmapherese auch Gerinnungsfaktoren entfernt, sind eine engmaschige Kontrolle des Gerinnungsstatus und ggf. auch die Gabe von FFP erforderlich (z. B. bei einer Fibrinogenkonzentration von $<100 \mathrm{mg} / \mathrm{dl}$ ), insbesondere bei Patienten mit alveolärer Hämorrhagie. Studiendaten zum Einsatz der Plasmapherese in der Rheumatologie existieren für die Kleingefäßvaskulitiden, das katastrophale Antiphospholipidantikörpersyndrom (CAPS) und den SLE. Ein weiteres Problem stellt die schlechte Abbildung des Verfahrens im DRG(Diagnosis Related Groups)-System dar. Der Erlös durch das Zusatzentgelt von rund $1250 €$, das für die Plasmaseparation abgerechnet werden darf, deckt nicht selten noch nicht einmal die notwendigen Humanalbuminmengen, geschweige denn den 
Einsatz von FFPs ab. An einen adäquaten Gegenwert von Geräteeinsatz und notwendigem Personal ist hierbei gar nicht zu denken. Dies führt dazu, dass das Austauschvolumen aus monetären Gründen häufig zu gering angesetzt und das Verfahren damit unterdosiert wird, wie eine retrospektive Auswertung gezeigt hat [61].

Eine Alternative zum Plasmaaustausch stellt die Immunadsorption dar, die mithilfe regenerierbarer immunologischer Säulen eine selektive IgGDepletion erzielt und damit die hämostaseologischen Komplikationen des kompletten Plasmaaustausches umgeht. Allerdings stellen ihr komplexer Aufbau und der hohe technische und personelle Aufwand sowie v. a. die geringe Evidenz erhebliche Limitationen dieses Verfahrens dar, und es ist spezialisierten Zentren vorbehalten.

\section{ANCA-assoziierte Vaskulitiden}

Die Untersuchung der Plasmapherese bei den ANCA-assoziierten Vaskulitiden (AAV) erfolgte primär unter der Annahme, dass ANCA für die Akutpathogenese der Erkrankung eine zentrale Rolle spielen [67]. Die Plasmapherese entfernt aber auch andere potenziell pathogenetisch relevante Plasmabestandteile [34], z.B. Gerinnungsfaktoren, was die Bildung von Mikrothromben und somit ischämische Organschäden reduzieren könnte [67]. Erste Hinweise auf eine mögliche Wirksamkeit der Plasmapherese bei den Kleingefäßvaskulitiden kamen in den 1970er- und 1980er-Jahren aus kleineren und in der Fallzahl unterpowerten Studien mit zudem heterogener immunsuppressiver Begleittherapie $[41,53]$. Die dann in 1990er-Jahren konzipierte MEPEX-Studie untersuchte den Stellenwert der Plasmapherese als Add-on-Therapie zu Cyclophosphamid (CYC) und GC im Vergleich zu $3 \mathrm{Me}-$ thylprednisolon-Pulsen zu Beginn der remissionsinduzierenden Therapie [29, 68]. Positive Signale zugunsten einer Wirksamkeit der Plasmapherese in der kurzfristigen Beobachtung [29] bestätigten sich im Langzeit-Follow-up nicht [68]. Bei insgesamt sehr hoher Mortalität, bedingt v. a. durch Infektionen,

Z Rheumatol 2019 · 78:955-966 https://doi.org/10.1007/s00393-019-00687-0

(c) Springer Medizin Verlag GmbH, ein Teil von Springer Nature 2019

B. Hellmich · C. Löffler

\section{Was sind die Indikationen für Rescue-Verfahren? Rheumatische Systemerkrankungen auf der Intensivstation}

\section{Zusammenfassung}

Schwere, organ- oder lebensbedrohliche Manifestationen entzündlicher rheumatischer Erkrankungen, wie z.B. eine diffuse alveoläre Hämorrhagie im Rahmen einer Kleingefäßvaskulitis, sprechen nicht immer ausreichend oder mit zeitlicher Verzögerung auf eine immunsuppressive Therapie an. Bei einem drohenden oder bereits eingetretenen Organversagen besteht dann nicht selten die Notwendigkeit, die immunsuppressive Therapie auf der Intensivstation um rasch wirksame Rescue-Therapieverfahren zu ergänzen. Aufgrund der Seltenheit vieler rheumatischer Erkrankungen ist die Evidenz zum Einsatz von Rescue-Therapieverfahren wie der Plasmapherese, der extrakorporalen Membranoxygenierung (ECMO) oder der Gabe von intravenösen Immunglobulinen (IVIG) für viele Indikationen eher gering. Der Einsatz der Plasmapherese wird bei einer akuten Anti-GBM(glomeruläre Basalmembran)-Erkrankung (Goodpasture Syndrom) oder einem katastrophalen Antiphospholipidantikörpersyndrom (CAPS) als sinnvoll angesehen. Eine ECMO-Therapie kann bei persistierender respiratorischer Insuffizienz trotz mechanischer Beatmung als Folge einer diffusen alveolären Hämorrhagie oder eines Acute-Respiratory-DistressSyndroms (ARDS) anderer Ursache erwogen werden. Eine Gabe von IVIG ist bei einer akuten kardialen Beteiligung im Rahmen einer Kawasaki-Erkrankung indiziert und kann beim CAPS sowie bei therapierefraktären Myositiden erwogen werden.

Schlüsselwörter

Plasmapherese · Intravenöse Immunglobuline Extrakorporale Membranoxygenierung . Vaskulitis $\cdot$ Kollagenose

\section{What are the indications for rescue procedures? Systemic rheumatic diseases in the intensive care unit}

\section{Abstract}

Severe, organ-threatening and lifethreatening manifestations of inflammatory rheumatic diseases, such as diffuse alveolar hemorrhage in the context of small vessel vasculitis, sometimes inadequately respond to immunosuppressive treatment. In the case of an immanent or already occurring organ failure, immunosuppressive treatment may need to be supplemented with rapidly effective rescue treatment procedures. Due to the rarity of many rheumatic diseases, the evidence for the use of rescue treatment, such as plasmapheresis, extracorporeal membrane oxygenation (ECMO) and the administration of intravenous immunoglobulins (IVIG), is relatively low for many indications. The use of plasmapheresis is considered useful in acute anti-glomerular basement membrane (GBM) disease (Goodpasture's syndrome) or catastrophic antiphospholipid antibody syndrome (APS). The use of ECMO treatment may be considered for persistent respiratory failure despite mechanical ventilation due to diffuse alveolar hemorrhage or acute respiratory distress syndrome (ARDS). Administration of IVIG is indicated for acute cardiac involvement in Kawasaki's disease and may be considered in catastrophic APS and refractory myositis.

\section{Keywords}

Plasmapheresis · Intravenous immunoglobulins - Extracorporeal membrane oxygenation . Vasculitis · Collagen vascular diseases wurde der primäre Endpunkt eines reduzierten Risikos für ein terminales Nierenversagen oder Tod nicht erreicht [68]. Vielmehr ergab sich ein nichtsignifikanter Trend für mehr infektionsbedingte Todesfälle. Eine Metaanalyse von noch vor Publikation dieser negativen Langzeitdaten durchgeführten Studien (einschließlich der frühen MEPEX-Da- ten) hatte zunächst noch Hinweise auf eine ca. 20\%ige Reduktion des Risikos für ein terminales Nierenversagen oder Tod ergeben, getrieben durch eine $36 \%$ ige Reduktion des Risikos für eine dialysepflichtige Niereninsuffizienz, die aber trotz formal statistischer Signifikanz nur wenig robust erschien, da bereits bei einer Abweichung um 2 klinische Ereig- 
Tab. 3 Indikationen zur Therapie mit intravenösen Immunglobulinen bei kritisch kranken Patienten mit entzündlich-rheumatischen Erkrankungen

\begin{tabular}{|c|c|c|c|}
\hline Erkrankung & Indikation & Evidenzgrad $^{\mathrm{a}}$ & Kommentar \\
\hline \multirow{2}{*}{$\begin{array}{l}\text { Immunthrombo- } \\
\text { zytopenie }\end{array}$} & Bei akuter Blutungskomplikation & $1 \mathrm{~b}$ & \multirow[t]{2}{*}{ In Kombination mit GC } \\
\hline & $\begin{array}{l}\text { Bei GC-refraktärer Thrombopenie vor Operatio- } \\
\text { nen oder bei Schwangeren }\end{array}$ & 4 & \\
\hline Kawasaki-Syndrom & Myokardinfarkt, akutes Koronarsyndrom & $1 b$ & First-Line-Therapie in Kombination mit ASS \\
\hline \multirow[t]{3}{*}{$\begin{array}{l}\text { ANCA- } \\
\text { assoziierte } \\
\text { Vaskulitis }\end{array}$} & $\begin{array}{l}\text { Therapierefraktäre Vaskulitis (ohne lebens- } \\
\text { oder organbedrohende Manifestation) }\end{array}$ & $1 b$ & $\begin{array}{l}\text { Second-Line-Therapie bei Versagen einer Standardin- } \\
\text { duktionstherapie mit GC plus CYC oder RTX oder } \\
\text { RTX/CYC-Kombination }\end{array}$ \\
\hline & $\begin{array}{l}\text { Aktive Vaskulitis + begleitende schwere Infekti- } \\
\text { on }\end{array}$ & 5 & \multirow[t]{2}{*}{$\begin{array}{l}\text { Cave: geringe Evidenz für Therapie schwerer } \\
\text { Organbeteiligungen }\end{array}$} \\
\hline & Sekundäre Hypogammaglobulinämie & 4 & \\
\hline \multirow[t]{3}{*}{$\begin{array}{l}\text { Inflammatorische } \\
\text { Myositiden }\end{array}$} & Refraktäre Myositis & $1 b$ & $\begin{array}{l}\text { Bei refraktärer Erkrankung trotz Therapie mit GC und anderen } \\
\text { Immunsuppressiva }\end{array}$ \\
\hline & Versagen der Atemmuskulatur & 4 & \multirow{2}{*}{$\begin{array}{l}\text { Wenig Evidenz für Wirkung auf interstitielle } \\
\text { Lungenerkrankung }\end{array}$} \\
\hline & Refraktäre Herzbeteiligung & 4 & \\
\hline \multirow[t]{2}{*}{$\begin{array}{l}\text { Katastrophales } \\
\text { APS }\end{array}$} & \multirow[t]{2}{*}{ Multiorganversagen } & \multirow[t]{2}{*}{4} & $\begin{array}{l}\text { In Kombination mit GC und Heparin, alternativ zur Plasma- } \\
\text { pherese }\end{array}$ \\
\hline & & & Leitlinienempfehlung \\
\hline
\end{tabular}

nisse in einer der Studien die Signifikanz verloren gegangen wäre [69].

\section{》) Die derzeit verfügbare Evidenz spricht gegen einen Einsatz der Plasmapherese bei Patienten mit AAV}

Die inkonsistenten Studiendaten, die in der Metaanalyse gesehene geringe Effektstärke der Plasmapherese und die später publizierten negativen Ergebnisse der MEPEX-Studie führten $\mathrm{zu}$ einer unterschiedlichen Bewertung des Stellenwertes der Plasmapherese durch die wissenschaftlichen Fachgesellschaften, die von einem regelhaften Einsatz bei schwerer AAV (British Society of Rheumatology) bis zu einer Empfehlung gegen einen Einsatz (Kanadische Vaskulitis Gesellschaft) reichten $[45,51$, 74]. In den DGRh(Deutsche Gesellschaft für Rheumatologie e. V.)-Leitlinien zum Management der AAV von 2017 ist die vorsichtige Empfehlung enthalten, den Einsatz der Plasmapherese bei aktiver Glomerulonephritis mit schwerer Einschränkung der Nierenfunktion zu erwägen [60].
Die kontroverse Bewertung des Stellenwertes der Plasmapherese in der Therapie der AAV führte zur PEXIVASStudie. Diese mit 702 randomisierten $\mathrm{Pa}$ tienten bis dato weltweit größte kontrollierte Studie untersuchte die Wirksamkeit der Plasmapherese als Add-on zu einer Standardinduktionstherapie mit Rituximab (RTX) oder CYC im Hinblick auf den kombinierten primären Endpunkt terminales Nierenversagen plus Mortalität [71]. Erste als Kongressabstract publizierte Ergebnisse zeigen, dass weder der primäre Endpunkt (HR [Hazard Ratio] 0,86 [95\%-KI (Konfidenzintervall) $0,65-1,13], p=0,027)$ noch die zahlreichen sekundären Endpunkte erreicht wurden [70]. Auch konnten in dieser ersten Analyse keine Subgruppen identifiziert werden (z.B. Patienten mit alveolärer Hämorrhagie oder schwerer Niereninsuffizienz), die von einer Plasmapherese profitieren [70]. Eine per Protokoll im Vergleich zum bis dato üblichen Standard schnellere GC-Reduktion auf eine Zieldosis von 7,5 statt $15 \mathrm{mg}$ nach Woche 12 führte nicht $\mathrm{zu}$ einem Wirkverlust, ging aber mit einer geringeren Rate schwerer Infektionen einher. Da das bereits im Volltext publizierte Studienprotokoll [71] keine relevanten methodischen Schwächen der Studie erkennen lässt und eine hohe Fallzahl erreicht wurde, können die ersten Ergebnisse als sehr robust angesehen werden. Zusammenfassend spricht die jetzt verfügbare Evidenz somit gegen einen Einsatz der Plasmapherese bei Patienten mit AAV.

\section{Anti-GBM-Erkrankung (Goodpasture-Syndrom)}

Die Anti-GBM(glomeruläre Basalmembran)-Erkrankung ist durch eine akute Immunkomplexglomerulonephritis charakterisiert, die sich häufig im Rahmen eines pulmorenalen Syndroms mit gleichzeitiger diffuser alveolärer Hämorrhagie präsentiert und durch ein akutes Nierenund Lungenversagen häufig eine intensivstationäre Therapie erforderlich macht [30,43]. Das Autoantigen der Anti-GBMAntikörper (eine Domäne der Alpha-3Kette von Typ-IV-Kollagen) wird insbesondere auf dem klinisch betroffenen Gewebe (Basalmembran der alveolären und glomerulären Kapillaren) exprimiert [11]. Da auch die Pathogenität der AntiGBM-Antikörper bereits 1967 in einem Tiermodell gezeigt werden konnte [38], ist der Einsatz der Plasmapherese theoretisch gut begründet und zielt auf eine 
Hier steht eine Anzeige.

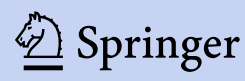


rasche Entfernung der pathogenen Antikörper aus der Zirkulation ab [43].

\section{) Entscheidend für die \\ Prognose ist v. a. die Nierenfunktion zum Zeitpunkt des Therapiebeginns}

Verschiedene Kohortenstudien konnten zeigen, dass eine rasche und intensive Therapie unter Einbezug der Plasmapherese, eine bei Therapiebeginn noch erhaltene Nierenfunktion und möglichst wenig tubulointerstitielle Fibrose in der Nierenbiopsie zentrale Voraussetzungen für gutes Langzeitoutcome sind [3, 15, 26, $39,66]$. Im Gegensatz zu den AAV liegen für die Anti-GBM-Erkrankung allerdings keine hochwertigen randomisierten Studien zum Einsatz der Plasmapherese vor. In einer kleinen offen randomisierten Studie an 17 Patienten konnte eine langfristige Dialysepflichtigkeit nach einer Plasmapherese (in Kombination mit CYC und GC) häufiger verhindert werden (bei 6 von 8 Patienten) als bei den Patienten (3 von 9), die nur immunsuppressivbehandelt wurden [31]. Erfahrungen mit der Plasmapherese beim AntiGBM-Syndrom liegen sonst nur aus einigen retrospektiven Kohortenstudien vor, die aufgrund des Fehlens einer Kontrollgruppe jedoch bezüglich der Wirksamkeit einer additiven Plasmapherese naturgemäß wenig aussagekräftig sind. In einer der bis dato größten Kohortenstudien zur Anti-GBM-Erkrankung war bei Patienten mit einer additiven Plasmapheresebehandlung kein relevanter Langzeitbenefit im Vergleich zur Standardimmunsuppression mit CYC und GC erkennbar, es bestand allerdings ein erheblicher Selection-Bias zum Einsatz der Plasmapherese [15]. Andere Kohortenstudien konnten zeigen, dass eine bereits bestehende Oligurie, viele Komorbiditäten und ein Patientenalter von über 60 Jahren die Wahrscheinlichkeit einer Erholung der Nierenfunktion reduzieren [3, 39]. Entscheidend für die Prognose ist aber v. a. die Nierenfunktion zum Zeitpunkt des Therapiebeginns. So konnte in einer Kohortenstudie nach kombinierter Therapie mit Plasmapherese, CYC und
GC bei einem Serumkreatinin von kleiner $500 \mu \mathrm{mol} / \mathrm{l}$ die Nierenfunktion nach 5 Jahren bei $95 \%$ erhalten werden, bei primärer Dialysepflichtigkeit aber nur bei $13 \%$ [39].

Bis zu $50 \%$ Patienten mit Anti-GBMErkrankung sind auch MPO- oder PR3ANCA-positiv [44]. Die Daten zur Prognose von ANCA-positiven Patienten mit Anti-GBM-Erkrankung sind uneinheitlich - es wurden sowohl eine höhere als auch niedrige Wahrscheinlichkeit eines Erhalts der Nierenfunktion bei positivem ANCA berichtet [3, 15, 44]. Ergebnisse einer Kohortenstudie an 28 Patienten konnten zeigen, dass eine Immunadsorption im Vergleich zu einer Doppelfiltrationsplasmapherese nicht zu einem besseren therapeutischen Ergebnis (Entfernung von Anti-GBM-Antikörper) führte, aber mit einem höheren Gesamt-IgGVerlust assoziiert war [75]. Die Leitlinien der KDIGO (Kidney Disease: Improving Global Outcomes) empfehlen, bei Patienten mit akuter Anti-GBM-Erkrankung zusätzlich zur Immunsuppression mit CYC (oral 2-3 mg/kg plus Prednisolon) eine Plasmapherese als First-LineTherapie durchzuführen, bis die AntiGBM-Antikörper im Serum nicht mehr nachweisbar sind [55]. Ausgenommen von dieser Empfehlung sind Patienten mit bereits bestehender Dialysepflichtigkeit oder fehlender alveolärer Hämorrhagie vor Therapiebeginn oder Patienten mit $100 \%$ Halbmonden in der Nierenbiopsie [55].

\section{Kryoglobulinämische Vaskulitis}

Die kryoglobulinämische Vaskulitis ist eine Immunkomplexvaskulitis kleiner Gefäße als Folge einer Präzipitation von Immunglobulinen bei Kälteexposition, die klinisch insbesondere durch eine Trias, bestehend aus Purpura, Arthritiden und eine Polyneuropathie, charakterisiert ist [30]. Schwere Organmanifestationen wie eine Glomerulonephritis und eine gastrointestinale Beteiligung oder ein Hyperviskositätssyndrom bei sehr hohem Kryokrit können Anlass für eine Aufnahme des Patienten auf die Intensivstation sein. Die Standardbehandlung der kryoglobulinämischen Vaskulitis besteht bei der häufig kausal assoziierten
Hepatitis C in einer heute in der Regel sehr effektiven antiviralen Therapie und bei der essenziellen Kryoglobulinämie in einer immunsuppressiven Therapie mit GC, je nach Krankheitsschwere ergänzt um CYC und andere Immunsuppressiva oder RTX [20, 56, 76].

Kryoglobuline können mit der Plasmapherese rasch aus der Zirkulation entfernt werden [59], sodass die Rationale zum Einsatz der Plasmapherese plausibel erscheint, wenn eine rasche und effektive Senkung der Kryoglobulinkonzentration erforderlich ist, z.B. bei unmittelbar drohendem oder bereits eingetretenem Organversagen trotz konservativer Therapie [58]. Qualitativ hochwertige Studien zum Einsatz der Plasmapherese bei der kryoglobulinämischen Vaskulitis existieren allerdings nicht. In einer kleinen randomisierten offenen Studie erhielten insgesamt 9 von 17 Patienten mit HCV(Hepatitis-C-Virus)-assoziierter kryoglobulinämischer Vaskulitis eine Plasmapheresetherapie zusätzlich zu einer individuell sehr unterschiedlichen Standardtherapie [64]. Ein klinischer Composite-Score verbesserte sich in beiden Armen um mehr als $50 \%$, lag zum Ende der Beobachtung bei den mit Plasmapherese behandelten Patienten aber niedriger als bei den nur konventionell therapierten Patienten $(10,0 \pm 0,7$ vs. $12,1 \pm 2,2, p=0,03)$ [64]. Aus einigen Fallserien mit 5 bis $23 \mathrm{~Pa}$ tienten wurde ein positives klinisches Ansprechen berichtet. Da die Patienten in der Regel zeitgleich mit verschiedenen Immunsuppressiva, GC und zum Teil auch antiviral behandelt wurden, bleibt im Ergebnis offen, welche Intervention im Einzelnen zum Therapieerfolg beigetragen hat. Zudem enthält die Mehrzahl der Fallserien weder Angaben zu Methode und Umfang der Plasmapheresebehandlungen noch Informationen zum Kurzzeitverlauf, die Rückschlüsse auf eine kurzfristige Wirksamkeit der Plasmapherese ermöglichen würden, wie z. B. einen raschen Abfall des Kryokrits oder Anstieg des typischerweise stark erniedrigten C4-Komplements. Eine Befragung von 22 Zentren aus Italien ergab, dass bei lediglich 83 von 159 mit Plasmapherese behandelten Patienten ein gutes oder sehr gutes Therapieansprechen 
beobachtet wurde [42]. Allerdings enthält auch diese retrospektive Befragung keine validen Angaben zur möglichen Wirksamkeit oder Ineffektivität einer begleitenden immunsuppressiven oder antiviralen Therapie. Die Internationale Studiengruppe zur extrahepatischen Manifestationen der Hepatitis C empfiehlt, dass eine Plasmapherese zusätzlich zur Standardtherapie bei schwerer/ lebensbedrohlicher HCV-assoziierter kryoglobulinämischer Vaskulitis oder einem Hyperviskositätssyndrom erwogen werden kann (Evidenzgrad 3, Empfehlungsstärke C) [56]. Zur essenziellen kryoglobulinämischen Vaskulitis existieren keine Leitlinienempfehlungen, in der klinischen Praxis scheint jedoch eine Indikationsstellung wie bei der HCVassoziierten Form sinnvoll zu sein.

\section{Andere Vaskulitiden}

Eine randomisierte kontrollierte Studie zum additiven Einsatz der Plasmapherese bei Patienten mit Polyarteriitis nodosa oder eosinophiler Granulomatose mit Polyangiitis wurde vorzeitig abgebrochen, nachdem eine Interimsanalyse der bis dahin behandelten 62 Patienten keine Verbesserung der 5-Jahres-Mortalität gezeigt hatte [24]. Zu anderen Vaskulitiden liegen keine Studiendaten vor.

\section{Systemischer Lupus erythematodes}

Ergebnisse einer randomisierten Studie bei 86 Patienten mit schwerer Lupusnephritis ergaben keine Hinweise auf eine Wirksamkeit einer Plasmapheresebehandlung (3-mal pro Woche über 4 Wochen) zusätzlich zur Standardtherapie. Zwar zeigte sich in der Plasmapheresegruppe ein rascherer Abfall der ds(doppelsträngige)DNA(Desoxyribonukleinsäure)-Anti-

körper, Mortalität und Nierenfunktion unterschieden sich aber nicht von der Kontrollgruppe [40]. Eine systematische Literaturanalyse zur Plasmapherese beim SLE ergab, dass die zahlreichen Fallserien zum Einsatz der Plasmapherese bei Patienten mit refraktärem SLE in der Summe widersprüchliche Ergebnisse zeigten [35]. Durch die gleichzeitige
Verabreichung von GC und hochpotenten Immunsuppressiva wie CYC ist der Anteil einer Plasmapherese am Therapieerfolg in diesen Studien ohne Kontrollgruppe kaum zu beurteilen. Diese Einschränkung gilt auch für die publizierten Fallserien zur Immunadsorption. In Anbetracht der negativen Ergebnisse der Lupusnephritisstudie einerseits und der zum Teil positiven Signalen aus offenen Studien zu refraktären Patienten andererseits wären kontrollierte Studien zum Einsatz der Plasmapherese oder Immunadsorption bei therapierefraktären SLE-Patienten wünschenswert. Ein mögliches Einsatzgebiet der Plasmapherese könnte in der Rescue-Therapie schwer betroffener schwangerer Patientinnen liegen, um hier den Einsatz von CYC und anderen potenziellen teratogenen Substanzen zu umgehen, Studiendaten $\mathrm{zu}$ diesem Indikationsgebiet fehlen aber ebenfalls [35].

\section{Katastrophales Antiphospholipid- syndrom}

Das katastrophale Antiphospholipidsyndrom (CAPS) ist ein komplexes klinisches Syndrom, das durch akut auftretende multifokale Thrombosen und resultierendes Multiorganversagen bei Patienten gekennzeichnet ist, welche die serologischen Kriterien für ein APS erfüllen [6]. Die McMaster RARE-Bestpractices Guidelines empfehlen einen First-LineEinsatz der Plasmapherese (als Alternative zu IVIG) bei CAPS als Teil einer Kombinationstherapie mit GC und Heparin [37]. Die aufgrund der formal niedrigen Evidenz als bedingt ausgesprochene Empfehlung basierte v. a. auf Ergebnissen einer Metaanalyse von Registerdaten, die eine reduzierte Mortalität unter einer solchen Kombinationsbehandlung zeigte, wobei der Effekt für die Plasmapherese als Einzelmaßnahme nicht signifikant war (OR [Odds Ratio] 0,68; $95 \%$ KI 0,41-1,12) [37]. Auffällig war eine signifikant höhere Rate an permanenten Organschädigungen und neurologischen Defiziten bei den mit Plasmapherese behandelten Patienten [37]. Bei gut einem Drittel der behandelten CAPS-Patienten wurden unter der Plasmapherese unerwünschte Ereignisse beobachtet, unter anderem Fieber (7,7\%), Urtikaria (7,4\%) und eine symptomatische Hypokalzämie $(7,3 \%)[63]$.

Die Empfehlungen der McMaster RARE-Bestpractices Guidelines zur Plasmapherese bei CAPS sind allerdings umstritten [52], da nur ein Teil der publizierten Literatur berücksichtigt wurde und kontrollierte Studien nicht vorliegen. Die Amerikanische Gesellschaft für Apherese empfiehlt die Plasmapherese daher nur als Zweitlinienverfahren [62]. Ein spezielles Problem der Plasmapherese bei Patienten mit APS stellt die Entfernung von Antikoagulanzien dar. So fällt z.B. nach einer Plasmapherese die Anti-Xa-Konzentration um ca. 50\% $a b$, was eine Erhöhung der verabreichten Heparindosierungen erforderlich macht [33].

\section{Makrophagenaktivierungssyndrom}

Kasuistisch ist der erfolgreiche Einsatz der Plasmapherese bei überwiegend pädiatrischen Patienten mit einem Makrophagenaktivierungssyndrom auf dem Boden einer juvenilen idiopathischen Arthritis, eines SLE, einer Dermatomyositis bzw. juvenilen Dermatomyositis beschrieben worden $[7,9,32]$.

\section{Extrakorporale Membran- oxygenierung}

Die extrakorporale Membranoxygenierung (ECMO) ist ein extrakorporales Therapieverfahren zur Unterstützung des Gasaustausches bei Patienten mit schwerer respiratorischer Insuffizienz trotz invasiver mechanischer Beatmung [8]. Die ECMO wird als Rescue-Therapie in ausgewählten intensivmedizinischen Zentren überwiegend bei Patienten mit Acute-Respiratory-Distress-Syndrom (ARDS) eingesetzt $[8,14]$. Studien speziell zur ECMO-Therapie bei Patienten mit rheumatologischen Systemerkrankungen existieren nicht. Die ECMO kann z. B. im Rahmen der allgemeinen intensivmedizinischen Behandlung bei (opportunistischen) Infektionen unter immunsuppressiver Therapie eingesetzt werden, z. B. bei einer schweren Pneumocystis-jirovecii-Pneumonie [4]. Eine weitere mögliche Indikation der ECMO 
bei Patienten mit rheumatologischen Systemerkrankungen stellt ein ARDS im Rahmen einer diffusen alveolären Hämorrhagie (DAH) bei Patienten mit Kleingefäßvaskulitiden dar, z.B. den AAV und der Anti-GBM-Erkrankung $[1,16,19]$. Zu beachten ist beim Einsatz der ECMO bei Patienten mit DAH die grundsätzliche Notwendigkeit zu einer Antikoagulation, um den ungestörten Blutfluss durch das ECMO-System zu gewährleisten. Die Antikoagulation erhöht theoretisch das Risiko einer Verschlechterung der DAH [1].

\section{\) Die ECMO wird als Rescue- Therapie bei Patienten mit ARDS eingesetzt}

Einheitliche Empfehlungen zur optimalen Antikoagulationsstrategie im Rahmen einer ECMO bei DAH existieren nicht. Eine ECMO-Therapie ohne Antikoagulation wurde in Einzelfällen als komplikationslos beschrieben [48], führt in anderen Fällen aber zu thrombotischen Komplikationen, die einen Austausch des ECMO-Systems erforderlich machten [1]. Eine kontinuierliche, aber möglichst niedrig dosierte Antikoagulation mit hohem Blutfluss im ECMOSystem (= geringeres Thromboserisiko im System) wurde in Einzelfällen mit DAH erfolgreich und ohne Zeichen einer Progredienz der pulmonalen Blutung eingesetzt [1]. Eine aktuelle systematische Analyse von Einzelfallberichten ergab, dass alle 14 untersuchten Patienten mit AAV und schwerer DAH den Krankenhausaufenthalt überlebten, die mittels ECMO, Immunsuppression und teils auch Plasmapheresen behandelt wurden [19]. Bei 2 der Patienten kam es zu schweren Blutungskomplikationen [19].

Studiendaten zum Einsatz der ECMO bei anderen entzündlich-rheumatischen Erkrankungen liegen nicht vor. Der Einsatz der ECMO kann jedoch bei Patienten mit einer interstitiellen Lungenerkrankung (ILD) im Rahmen einer RA (RA-ILD) oder Kollagenose (CTDILD) und akuter respiratorischer Insuffizienz erwogen werden, jedoch nur, wenn der Patient kurzfristig für eine Lungentransplantation vorgesehen ist oder eine behandelbare akute Komplikation (z.B. schwere Pneumonie) bei einem Patienten auftritt, dessen ILD noch nicht weit fortgeschritten ist (ausreichender Oxygenierung vor dem Ereignis) [47]. In einer retrospektiven Fallserie von $42 \mathrm{~Pa}$ tienten mit unterschiedlichen Formen einer überwiegend nicht CTD- oder RAassoziierten ILD und akutem respiratorischem Versagen verstarben $93 \%$ der Patienten trotz ECMO-Therapie, wenn diese nicht lungentransplantiert wurden [65]. Fünf von 6 Patienten, die während des stationären Aufenthaltes ein Lungentransplantat erhielten, überlebten unter Einsatz der ECMO als Überbrückungsmaßnahme.

\section{Intravenöse Immunglobuline}

Die Evidenz zur Therapie mit intravenösen Immunglobulinen (IVIG) in der Rheumatologie wurde von T. Witte in dieser Zeitschrift kürzlich umfassend dargestellt [73], sodass für eine Darstellung der Studien zu den einzelnen Krankheitsbildern auf diese Publikation verwiesen wird. Kontrollierte Studien zum Einsatz von IVIG als Rescue-Verfahren speziell bei kritisch kranken Patienten mit entzündlich-rheumatischen Erkrankungen auf der Intensivstation existieren nicht.

\section{Immunthrombozytopenie}

Die Gabe von IVIG kann in Analogie zur idiopathischen Immunthrombozytopenie (ITP) auch bei Patienten mit schwerer und therapierefraktärer Autoimmunthrombozytopenie im Rahmen eines SLE erwogen werden. Die Gabe von IVIG wird in Kombination mit GC bei akuten Blutungskomplikationen im Rahmen einer ITP empfohlen $[23,49]$ und ist in dieser Indikation auch zugelassen. Auch vor operativen Eingriffen oder bei Schwangeren mit ITP kann ein Einsatz von IVIG erwogen werden, wenn GC nicht ausreichend wirksam waren oder vermieden werden müssen [73].

\section{Vaskulitiden}

Für die AAV liegen Ergebnisse einer kleinen randomisierten placebokontrollierten Studie bei Patienten mit Restaktivität unter eine Therapie mit CYC und GC vor [28]. Ein Therapieansprechen (definiert als Rückgang des Birmingham Vasculitis Activity Score [BVAS] um $50 \%$ und mehr) wurde in der IVIG-Gruppe häufiger erreicht als in der Placebo-Gruppe [28]. Wohl bedingt durch die kleine Fallzahl zeigten sich keine Unterschiede bezüglich Mortalität, schweren unerwünschten Wirkungen und Zeit bis zum ersten Rezidiv - der Stellenwert von IVIG in der Therapie der AAV ist daher umstritten [22]. Zudem waren Patienten mit schweren Organbeteiligungen in der Studie ausgeschlossen, sodass die Studie für die Fragestellung einer Rescue-Therapie auf der Intensivstation nur eine indirekte Evidenz liefert. In der Praxis kann eine Off-Label-Gabe von IVIG als RescueTherapie bei AAV in Situationen erwogen werden, in denen eine hochpotente Induktionstherapie mit hoch dosierten GC und CYC oder RTX vermieden werden soll, z. B. aufgrund einer parallel vorliegenden Infektion.

Eine intensivstationäre Behandlung kann bei Patienten mit einem KawasakiSyndrom z.B. aufgrund eines akuten Myokardinfarkts erforderlich sein. Die Gabe von IVIG gilt zusammen mit einer Verabreichung von ASS als Therapie der ersten Wahl und ist, basierend auf den Ergebnissen einer randomisierten kontrollierten Studie, in dieser Indikation auch formal zugelassen [50].

\section{》) Beim Kawasaki-Syndrom ist IVIG zusammen mit einer Verabreichung von ASS Therapie der ersten Wahl}

$\mathrm{Zu}$ anderen Vaskulitiden wie der IgAVaskulitis oder Polyarteriitis nodosa liegen nur wenige Einzelfallberichte vor, die keine höherwertige Evidenz zum Stellenwert der Therapie bei kritischen Organmanifestation liefern [5]. Bei der kryoglobulinämischen Vaskulitis wird eine IVIG-Therapie nicht empfohlen, da das IgG mit den in den Kryoglobulinen gebundenen IgM-Antikörpern Immunkomplexe bilden kann [73]. 


\section{Kollagenosen}

Die Wirksamkeit einer IVIG-Therapie ist bei den inflammatorischen Myositiden durch Daten einer randomisierten kontrollierten Studie belegt [17]. Für Patienten mit schweren, teils auch lebensbedrohlichen Manifestation (z. B. Herzbeteiligung oder Dysphagie) liegen Erfahrungen aus einer offenen Studie an 7 Patienten vor [18]. Eine Wirksamkeit von IVIG auf die häufig prognosebestimmende interstitielle Lungenerkrankung ist weniger gut dokumentiert. Die IVIG-Therapie kann aber bei respiratorischem Versagen als Folge einer Insuffizienz der Atemmuskulatur erwogen werden [72]. IVIG sind zur Therapie der Myositiden formal nicht zugelassen, können aber laut Beschluss des gemeinsamen Bundesausschusses bei therapierefraktärer Poly- oder Dermatomyositis als Rescue-Therapie additiv zur Gabe von GC und Azathioprin verordnet werden [73].

IVIG werden als Alternative zu einer Plasmapherese auch im Rahmen ei- nes multimodalen Therapiekonzeptes in Kombination mit hoch dosierten GC und Heparin als First-Line-Therapie („offlabel“) beim katastrophalen APS empfohlen [37]. Kontrollierte Studien zum Einsatz von IVIG beim APS existieren nicht. Eine Metaanalyse von Patienten aus Registern und Fallserien ergab einen nichtsignifikanten Trend $\mathrm{zu}$ einer reduzierten Mortalität [37]. Kontrollierte Studien zum Einsatz von IVIG beim SLE, Sjögren-Syndrom und OverlapSyndrom liegen nicht vor. Speziell zur IVIG-Therapie bei organ- oder lebensbedrohlichen Manifestationen dieser Kollagenosen (z.B. zerebrale Beteiligung, schwere Lupusnephritis) findet sich in der Literatur nur wenig Evidenz. Zudem scheint eine IVIG-Therapie bei diesen Indikationen auch aufgrund der heute zur Verfügung stehenden medikamentösen Therapieoptionen (MycophenolatMofetil, Tacrolimus, Biologika wie Rituximab, Teilnahme an Studien ...) in der Regel entbehrlich zu sein.

\section{Andere Indikationen}

Bei Patienten mit primärer oder sekundärer Hypogammaglobulinämie im Rahmen einer rheumatischen Systemerkrankung, z.B. nach Therapie mit Rituximab, die aufgrund einer schweren Infektion intensivmedizinisch behandelt werden müssen, scheint eine IVIG-Gabe zur Akuttherapie des Immundefektes sinnvoll. In einer Serie von 12 Patienten mit AAV und sekundärem IgG-Mangel nach einer meist längeren Therapie mit RTX konnte durch eine IVIG-Gabe das Infektionsrisiko gesenkt werden [57]. Eine moderate Evidenz besteht zudem für den Einsatz von IVIG beim refraktären Pyoderma gangraenosum [73] sowie bei schweren Hautinfektionen wie der nekrotisierenden Fasziitis, dem Stevens-Johnson-Syndrom und dem Toxic-Shock-Syndrom [72]. Gesicherte Indikationen für eine IVIGTherapie bei kritisch kranken Patienten sind die Myasthenia gravis, das LambertEaton-Syndrom und das Guillan-Barré-

Hier steht eine Anzeige. 
Syndrom [72], die als Komorbiditäten bestehen können. In Fallserien ist zudem eine Wirksamkeit von IVIG bei Patienten mit einem Makrophagenaktivierungssyndrom beschrieben worden [2, 36].

\section{Fazit für die Praxis}

- Die formale Evidenz zum Einsatz von Rescue-Verfahren bei organ- oder lebensbedrohlichen Manifestationen ist für die Mehrzahl der entzündlichrheumatischen Erkrankungen gering. Nur wenige Therapien wie die IVIG(intravenöse Immunglobuline)Therapie beim Kawasaki-Syndrom sind formal zugelassen.

- Die Indikation für eine Plasmapherese oder IVIG-Therapie bei kritisch kranken Patienten sollte in Anbetracht der geringen Evidenz, möglichen Risiken (Plasmapherese) und hohen Kosten individuell abgewogen werden. Eine IVIG-Therapie oder Plasmapherese kann v. a. dann erwogen werden, wenn trotz schwerer akuter Organbeteiligungen eine aggressive immunsuppressive Therapie vermieden werden sollte, $\mathbf{z}$. $B$. bei gleichzeitig bestehender schwerer Infektion oder Schwangerschaft.

- Eine ECMO(extrakorporale Membranoxygenierung)-Therapie bei schwerer respiratorischer Insuffizienz trotz invasiver Beatmung sollte in erfahrenen Zentren durchgeführt werden.

\section{Korrespondenzadresse}

\section{Prof. Dr. med. B. Hellmich}

Vaskulitiszentrum Süd, Klinik für Innere Medizin, Rheumatologie und Immunologie, Medius Kliniken - Akademisches Lehrkrankenhaus, Universität Tübingen

Eugenstr. 3, 73230 Kirchheim u. Teck, Deutschland

b.hellmich@medius-kliniken.de

\section{Einhaltung ethischer Richtlinien}

Interessenkonflikt. B. Hellmich hat Honorare für Beratungsleistungen oder für Vorträge von Abbvie, BMS, Chugai, Pfizer, InflaRx und Roche erhalten. C. Löffler gibt an, dass kein Interessenkonflikt besteht.
Für diesen Beitrag wurden von den Autoren keine Studien an Menschen oder Tieren durchgeführt. Für die aufgeführten Studien gelten die jeweils dort angegebenen ethischen Richtlinien.

\section{Literatur}

1. Abrams D, Agerstrand CL, Biscotti M et al (2015) Extracorporeal membrane oxygenation in the management of diffuse alveolar hemorrhage. ASAIO J 61:216-218

2. Ahn JS, Rew SY, Shin MG et al (2010) Clinical significance of clonality and Epstein-Barr virus infection in adult patients with hemophagocytic lymphohistiocytosis. Am J Hematol 85:719-722

3. Alchi B, Griffiths M, Sivalingam M et al (2015) Predictors of renal and patient outcomes in antiGBM disease: clinicopathologic analysis of a twocentre cohort. Nephrol Dial Transplant 30:814-821

4. Ali HS, Hassan IF, George S (2016) Extra corporeal membrane oxygenation to facilitate lung protective ventilation and prevent ventilator-induced lung injury in severe pneumocystis pneumonia with pneumomediastinum: a case report and short literature review. BMC Pulm Med 16:52

5. Aries PM, Hellmich B, Gross WL (2005) Intravenous immunoglobulin therapy in vasculitis: speculation or evidence? Clin Rev Allergy Immunol 29:237-245

6. Asherson RA, Cervera R, De Groot PG et al (2003) Catastrophic antiphospholipid syndrome: international consensus statement on classification criteria and treatment guidelines. Lupus 12:530-534

7. Aytac S, Batu ED, Unal S et al (2016) Macrophage activation syndrome in children with systemic juvenile idiopathic arthritis and systemic lupus erythematosus. Rheumatol Int 36:1421-1429

8. Brodie D, Bacchetta M (2011) Extracorporeal membrane oxygenation for ARDS in adults. N Engl JMed 365:1905-1914

9. Bustos BR, Carrasco AC, Toledo RC (2012) Plasmapheresis for macrophage activation syndrome and multiorgan failure as first presentation of juvenile dermatomyositis. An Pediatr (Barc) 77:47-50

10. Camargo JF, Tobon GJ, Fonseca N et al (2005) Autoimmune rheumatic diseases in the intensive care unit: experience from a tertiary referral hospital and review of the literature. Lupus 14:315-320

11. Cashman SJ, Pusey CD, Evans DJ (1988) Extraglomerular distribution of immunoreactive Goodpasture antigen. JPathol 155:61-70

12. Cavallasca JA, Del Rosario Maliandi M, Sarquis $S$ et al (2010) Outcome of patients with systemic rheumatic diseases admitted toa medical intensive care unit. JClin Rheumatol 16:400-402

13. Clark WF, Huang SS, Walsh MW et al (2016) Plasmapheresis for the treatment of kidney diseases. Kidney Int 90:974-984

14. Combes A, Hajage D, Capellier G et al (2018) Extracorporeal membrane oxygenation for severe acute respiratory distress syndrome. N Engl J Med 378:1965-1975

15. Cui Z, Zhao J, Jia XY et al (2011) Anti-glomerular basement membrane disease: outcomes of different therapeutic regimens in a large singlecenter Chinese cohort study. Medicine (Baltimore) 90:303-311

16. Dalabih A, Pietsch J, Jabs K et al (2012) Extracorporeal membrane oxygenation as a platform for recovery: a case report of a child with pulmonary hemorrhage, refractory hypoxemic respiratory failure, and new onset goodpasture syndrome. JExtra Corpor Technol 44:75-77

17. Dalakas MC, Illa I, Dambrosia JM et al (1993) A controlled trial of high-dose intravenous immune globulin infusions as treatment for dermatomyositis. NEngl J Med 329:1993-2000

18. Danieli MG, Calcabrini L, Calabrese V et al (2009) Intravenous immunoglobulin as add on treatment with mycophenolate mofetil in severe myositis. Autoimmun Rev 9:124-127

19. Delvino P, Monti S, Balduzzi Setal (2019) The role of extra-corporeal membrane oxygenation (ECMO) in the treatment of diffuse alveolar haemorrhage secondary to ANCA-associated vasculitis: report of two cases and review of the literature. Rheumatol Int 39:367-375

20. Desbois AC, Comarmond C, Saadoun Det al (2017) Cryoglobulinemia vasculitis: how to handle. Curr Opin Rheumatol 29:343-347

21. Duru N, Van Der Goes MC, Jacobs JW et al (2013) EULAR evidence-based and consensusbased recommendations on the management of medium to high-dose glucocorticoid therapy in rheumatic diseases. Ann Rheum Dis 72:1905-1913

22. Fortin PM, Tejani AM, Bassett K et al (2013) Intravenous immunoglobulin as adjuvant therapy for Wegener's granulomatosis. Cochrane Database Syst Rev 1:Cd7057

23. Grainger JD, Bolton-Maggs $\mathrm{PH}$, Godeau $\mathrm{B}$ et al (2010) Diagnosis and management of chronic ITP: comments from an ICIS expert group. Ann Hematol 89(Suppl 1):11-17

24. Guillevin L, Fain O, Lhote F et al (1992) Lack of superiority of steroids plus plasma exchange to steroids alone in the treatment of polyarteriitis nodosa and Churg-Strauss syndrome. A prospective, randomized trial in 78 patients. Arthritis Rheum 35:208-215

25. Hollander D, Manning R (1967) The use of alcylating agents in the treatment of Wegener's granulomatosis. Ann Intern Med 67:393-398

26. Huart A, Josse AG, Chauveau D et al (2016) Outcomes of patients with Goodpasture syndrome: a nationwide cohort-based study from the French Society of Hemapheresis. J Autoimmun 73:24-29

27. Janssen NM, Karnad DR, Guntupalli KK (2002) Rheumatologic diseases in the intensive care unit: epidemiology, clinical approach, management, and outcome. Crit Care Clin 18:729-748

28. Jayne DR, Chapel H, Adu D et al (2000) Intravenous immunoglobulin for ANCA-associated systemic vasculitis with persistent disease activity. QJM 93:433-439

29. Jayne DR, Gaskin G, Rasmussen N et al (2007) Randomized trial of plasma exchange or highdosage methylprednisolone as adjunctive therapy for severe renal vasculitis. J Am Soc Nephrol $18: 2180-2188$

30. Jennette JC, Falk RJ, Bacon PA et al (2013) 2012 revised international Chapel Hill consensus conference nomenclature of vasculitides. Arthritis Rheum 65:1-11

31. Johnson JP, Moore J Jr., Austin HA 3rd et al (1985) Therapy of anti-glomerular basement membrane antibody disease: analysis of prognostic significance of clinical, pathologic and treatment factors. Medicine (Baltimore) 64:219-227

32. Kaieda S, Yoshida N, Yamashita F et al (2015) Successful treatment of macrophage activation syndrome in a patient with dermatomyositis by combination with immunosuppressive therapy and plasmapheresis. Mod Rheumatol 25:962-966

33. Kaplan A, Raut P, Totoe G et al (2016) Management of systemic unfractionated heparin anticoagu- 
lation during therapeutic plasma exchange. J Clin Apher 31:507-515

34. Keller AJ, Urbaniak SJ (1978) Intensive plasma exchange on the cell separator: effects on serum immunoglobulins and complement components. Br JHaematol 38:531-540

35. Kronbichler A, Brezina B, Quintana LF et al (2016) Efficacy of plasma exchange and immunoadsorption in systemic lupus erythematosus and antiphospholipid syndrome: a systematic review. Autoimmun Rev 15:38-49

36. Larroche C, Bruneel F, Andre MH et al (2000) Intravenously administered gamma-globulins in reactive hemaphagocytic syndrome. Multicenter study to assess their importance, by the immunoglobulins group of experts of CEDIT of the AP-HP. Ann Med Interne (Paris) 151:533-539

37. Legault K, Schunemann H, Hillis C et al (2018) McMaster RARE-Bestpractices clinical practice guideline on diagnosis and management of the catastrophic antiphospholipid syndrome. J Thromb Haemost. https://doi.org/10.1111/jth. 14192

38. Lerner RA, Glassock RJ, Dixon FJ (1967) The role of anti-glomerular basement membrane antibody in the pathogenesis of human glomerulonephritis. JExpMed 126:989-1004

39. Levy JB, Turner AN, Rees AJ et al (2001) Longterm outcome of anti-glomerular basement membrane antibody disease treated with plasma exchange and immunosuppression. Ann Intern Med 134:1033-1042

40. Lewis EJ, Hunsicker LG, Lan SP et al (1992) A controlled trial of plasmapheresis therapy in severe lupus nephritis. The Lupus Nephritis Collaborative Study Group. N Engl J Med 326:1373-1379

41. Lockwood CM, Rees AJ, Pearson TA et al (1976) Immunosuppression and plasma-exchange in the treatment of Goodpasture's syndrome. Lancet 1:711-715

42. Marson P, Monti G, Montani Fet al (2018) Apheresis treatment of cryoglobulinemic vasculitis: a multicentre cohort study of 159 patients. Transfus Apher Sci 57:639-645

43. Mcadoo SP, Pusey CD (2017) Anti-glomerular basement membrane disease. Clin J Am Soc Nephrol 12:1162-1172

44. McadooSP, TannaA,HruskovaZetal(2017)Patients double-seropositive for ANCA and anti-GBM antibodies have varied renal survival, frequency of relapse, and outcomes compared to singleseropositive patients. Kidney Int 92:693-702

45. Mcgeoch L, Twilt M, Famorca L et al (2016) CanVasc recommendations for the management of antineutrophil cytoplasm antibody-associated vasculitides. JRheumatol 43:97-120

46. Medicine OCFE-B (2009) Oxford Centre for Evidence-based Medicine-Levels of Evidence (March 2009). https://www.cebm.net/2009/06/ oxford-centre-evidence-based-medicine-levelsevidence-march-2009/.Zugegriffen: 8. Aug 2019

47. Moerer O, Quintel M (2016) Bridge or abyss: extracorporeal membrane oxygenation for acute respiratory failure in interstitial lung disease. Am J Respir Crit Care Med 193:474-476

48. Muellenbach RM, Kredel M, Kunze E et al (2012) Prolonged heparin-free extracorporeal membrane oxygenation in multiple injured acute respiratory distress syndrome patients with traumatic brain injury. J Trauma Acute Care Surg 72:1444-1447

49. Neunert CE, Cooper N (2018) Evidence-based management of immune thrombocytopenia: ASH guideline update. Hematology Am Soc Hematol Educ Program 2018:568-575
50. Newburger JW, Takahashi M, Burns JC et al (1986) The treatment of Kawasaki syndrome with intravenous gamma globulin. N Engl J Med 315:341-347

51. Ntatsaki E, Carruthers D, Chakravarty Ket al (2014) BSR and BHPR guideline for the management of adults with ANCA-associated vasculitis. Rheumatol (Oxford) 53:2306-2309. https://doi.org/10.1093/ rheumatology/ket445

52. Peedin AR, Karp JK (2019) Revisiting the role of therapeutic plasma exchange in the management of catastrophic antiphospholipid syndrome. J Thromb Haemost. https://doi.org/10.1111/jth. 14400

53. Pinching AJ, Lockwood CM, Pussell BA et al (1983) Wegener's granulomatosis: observations on 18 patients with severe renal disease. Q J Med 52:435-460

54. Quintero OL, Rojas-Villarraga A, Mantilla RD et al (2013) Autoimmune diseases in the intensive care unit. An update. Autoimmun Rev 12:380-395

55. Radhakrishnan J, Cattran DC (2012) The KDIGO practice guideline on glomerulonephritis: reading between the (guide)lines-application to the individual patient. Kidney Int 82:840-856

56. Ramos-Casals $M$, Zignego AL, Ferri $C$ et al (2017) Evidence-based recommendations on the management of extrahepatic manifestations of chronic hepatitis C virus infection. J Hepatol 66:1282-1299

57. Roberts DM, Jones RB, Smith RM et al (2015) Immunoglobulin $\mathrm{G}$ replacement for the treatment of infective complications of rituximab-associated hypogammaglobulinemia in autoimmune disease: A case series. J Autoimmun 10:168-161

58. Rockx MA, Clark WF (2010) Plasma exchange for treating cryoglobulinemia: a descriptive analysis. Transfus Apher Sci 42:247-251

59. Scarpato S, Tirri E, Naclerio C et al (2007) Plasmapheresis in cryoglobulinemic neuropathy: a clinical study. Dig Liver Dis 39(Suppl 1):S136-S137

60. Schirmer JH, Aries PM, De Groot K et al (2017) S 1 Leitlinie zur Diagnose und Therapie der ANCAassoziierten Vaskulitiden. ZRheumatol 76:77-104

61. Schmidt JJ, Asper F, Einecke G et al (2018) Therapeutic plasma exchange in a tertiary care center: 185 patients undergoing 912 treatments-a oneyear retrospective analysis. BMC Nephrol 19:12

62. Schwartz J, Padmanabhan A, Aqui N et al (2016) Guidelines on the use of therapeutic apheresis in clinical practice-evidence-based approach from the writing committee of the American Society for Apheresis: the seventh special issue. J Clin Apher 31:149-162

63. Shemin D, Briggs D, Greenan M (2007) Complications of therapeutic plasma exchange: a prospective study of 1,727 procedures. J Clin Apher 22:270-276

64. Stefanutti C, Vivenzio A, Di Giacomo S et al (2009) Immunoadsorption apheresis and immunosuppressive drug therapy in the treatment of complicated HCV-related cryoglobulinemia. JClin Apher 24:241-246

65. Trudzinski FC, Kaestner F, Schafers $\mathrm{HJ}$ et al (2016) Outcome of patients with interstitial lung disease treated with extracorporeal membrane oxygenation for acute respiratory failure. Am J Respir Crit Care Med 193:527-533

66. Van Daalen EE, Jennette JC, Mcadoo SP et al (2018) Predicting outcome in patients with antiGBM glomerulonephritis. Clin J Am Soc Nephrol 13:63-72
67. Walsh M (2014) Plasma exchange in antineutrophil cytoplasm antibody-associated vasculitis. Curr Opin Nephrol Hypertens 23:555-559

68. Walsh M, Casian A, Flossmann O et al (2013) Longterm follow-up of patients with severe ANCAassociated vasculitis comparing plasma exchange to intravenous methylprednisolone treatment is unclear. Kidney Int 84:397-402. https://doi.org/10. 1038/ki.2013.131.

69. Walsh M, Catapano F, Szpirt W et al (2011) Plasma exchange for renal vasculitis and idiopathic rapidly progressive glomerulonephritis: a meta-analysis. Am JKidney Dis 57:566-574

70. Walsh M, Merkel PA, Jayne D (2018) The effects of plasma exchange and reduced-dose glucocorticoids during remission induction for treatment of severe ANCA-associated vasculitis. Arthritis Rheumatol 70(suppl 10). https:// acrabstracts.org/abstract/the-effects-of-plasmaexchange-and-reduced-dose-glucocorticoidsduring-remission-induction-for-severe-ancaassociated-vasculitis/.Zugegriffen: 8. August 2019

71. Walsh M, Merkel PA, Peh CA et al (2013) Plasma exchange and glucocorticoid dosing in the treatment of anti-neutrophil cytoplasm antibody associated vasculitis (PEXIVAS): protocol for a randomized controlled trial. Trials 14:73

72. Wang J, Mcquilten ZK, Wood EM et al (2015) Intravenous immunoglobulin in critically ill adults: When and what is the evidence? J Crit Care 30(652):e659-e616

73. Witte T (2016) Therapeutic administration of immunoglobulins. ZRheumatol 75:956-963

74. Yates M, Watts RA, Bajema IM et al (2016) EULAR/ERA-EDTA recommendations for the management of ANCA-associated vasculitis. Ann Rheum Dis 75:1883-1894

75. Zhang YY, Tang Z, Chen DM et al (2014) Comparison of double filtration plasmapheresis with immunoadsorption therapy in patients with anti-glomerular basement membrane nephritis. BMC Nephrol 15:128

76. Zignego $A L$, Ramos-Casals $M$, Ferri $C$ et al (2017) International therapeutic guidelines for patients with HCV-related extrahepatic disorders. A multidisciplinary expert statement. Autoimmun Rev 16:523-541 
Hier steht eine Anzeige.

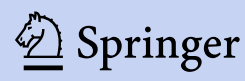

